\title{
Alcohol and oestrogen metabolites in postmenopausal women in the Women's Health Initiative Observational Study
}

\author{
Mary C Playdon ${ }^{*}{ }^{1}$, Sally B Coburn ${ }^{1}$, Steven C Moore ${ }^{1}$, Louise A Brinton ${ }^{1}$, Nicolas Wentzensen ${ }^{1}$, \\ Garnet Anderson ${ }^{2}$, Robert Wallace ${ }^{3}$, Roni T Falk ${ }^{1}$, Ruth Pfeiffer ${ }^{1}$, Xia Xu ${ }^{4}$ and Britton Trabert ${ }^{1}$ \\ ${ }^{1}$ Division of Cancer Epidemiology and Genetics, National Cancer Institute, 6909 Medical Centre Drive, Rockville, MD 20850, USA; \\ ${ }^{2}$ Public Health Sciences Division, University of Washington, 1100 Fairview Ave. N, M3-C102, Seattle, WA 98109, USA; ${ }^{3}$ College of \\ Public Health, The University of lowa, 145 N. Riverside drive, 100 CPHB, lowa City, IA 52242, USA and ${ }^{4}$ Hormone Analysis Unit, \\ Protein Characterization Laboratory, Cancer Research Technology Program, Leidos Biomedical Research Inc., P.O. Box B, \\ Frederick, MD 21702-1201, USA
}

Background: Alcohol consumption is associated with an increased risk of several cancers. Potential mechanisms include altered oestrogen metabolism. Parent oestrogens metabolise into alternate pathways of oestrogen metabolites that may have variable effects on cancer pathogenesis. We examined associations of alcohol consumption with circulating oestrogen/oestrogen metabolites in postmenopausal women in the Women's Health Initiative (WHI)-Observational Study (OS).

Methods: We conducted a cross-sectional analysis of prediagnosis ovarian/endometrial cancer case-control data within WHI-OS ( $N=1864)$. Alcohol consumption was measured by validated food frequency questionnaire. Fasting serum parent oestrogens/ oestrogen metabolites were assayed using liquid chromatography tandem mass-spectrometry. Geometric mean analyte concentrations $\left(\mathrm{GM}, \mathrm{pmol}^{-1}\right.$ ) were calculated by alcohol category using inverse-probability weighted linear regression, adjusting for venepuncture age/year, race, smoking, body mass index, years since menopause, oral contraceptive duration, caffeine intake, and physical activity.

Results: There was evidence for a positive association between alcohol consumption and oestrone, oestradiol and 2hydroxylation oestrogen metabolite concentrations among menopausal hormone therapy (MHT) users. We observed an association between liquor consumption and parent oestrogens among non-MHT users, who consumed larger doses of liquor than MHT users.

Conclusions: Among postmenopausal women, the association between alcohol intake and parent oestrogen, but not oestrogen metabolite concentrations, may be influenced by MHT and type of alcohol.

Alcohol consumption is ubiquitous across the globe, particularly among developed countries. There is convincing evidence that alcohol increases risk for breast cancer with a dose-response starting at low intake levels (World Cancer Research Fund/ American Institute for Cancer Research, 2010; Schutze et al, 2011; Jung et al, 2015). Alcohol may affect breast cancer risk through several potential mechanisms including acetaldehyde promotion of tumour initiation, ethanol metabolism-induced oxidative stress and tumour promotion, altered metabolism and clearance of carcinogens, and impaired immunity, as well as increased circulating sex steroid hormones, in part through aromatisation of androgens to oestrogens (IARC, 2010; Key et al, 2011).

*Correspondence: Dr MC Playdon; E-mail: mary.playdon@nih.gov

Received 27 June 2017; revised 25 October 2017; accepted 26 October 2017; published online 12 December 2017

(C) 2018 Cancer Research UK. All rights reserved 0007 - 0920/18 
Evidence from large, pooled analyses of prospective studies (Key et al, 2011) and several randomised-controlled crossover feeding studies (Dorgan et al, 2001; Mahabir et al, 2004; Sierksma et al, 2004) that used $95 \%$ ethanol as the alcohol exposure has shown that alcohol consumption is associated with higher circulating parent oestrogens (oestradiol, oestrone), androstenedione, dehydroepiandrosterone sulphate (DHEAS), testosterone, and lower sex hormone binding globulin (SHBG). Parent oestrogens can be irreversibly hydroxylated at steroid ring carbon positions 2 , 4 , or 16 to yield 2-, 4- or 16-hydroxylated oestrogen metabolites (Jefcoate et al, 2000). These pathways are interrelated, with metabolite concentrations within each pathway dependent on the overall oestrogen substrate pool. The mitogenic and genotoxic properties of oestrogen metabolites that affect cancer risk have been shown to differ by oestrogen metabolic pathway, partly influenced by degree of hydroxylation and/or methylation (Cavalieri et al, 2006; Yager, 2015; Cavalieri and Rogan, 2016; Dallal et al, 2016; Sampson et al, 2016). For example, in prospective studies utilising liquid chromatography-tandem mass spectrometry (LC-MS/MS) to measure oestrogen/oestrogen metabolites, relative increase in levels of 2-hydroxylation pathway metabolites to total has been associated with reduced breast cancer risk (Sampson et al, 2016). Oestrogen metabolism is consequently a highly complex hormonal exposure characterised by competing metabolic pathways. To understand the potential effects of alcohol on the hormonal mechanisms that drive cancer risk, a better understanding of downstream metabolic influences is needed.

Studies that explore the relationship between alcohol intake and oestrogen metabolism that include oestrogen pathway metabolites are limited (Hartman et al, 2016), and none, to our knowledge, have been conducted among postmenopausal women. Our objective was to examine associations of circulating serum oestrogen/oestrogen metabolite concentrations (in conjugated and unconjugated forms) with alcohol consumption, stratified by menopausal hormone therapy (MHT) use, in postmenopausal women participating in a nested case-control study within the Women's Health Initiative (WHI) Observational Study (OS). We hypothesised that alcohol drinkers would have higher parent oestrogens and oestrogen metabolites, and lower 2-pathway metabolites, favouring a pro-oestrogenic effect of alcohol with metabolism of parent oestrogen into 4- and 16-pathway compared with the 2-pathway metabolites.

\section{MATERIALS AND METHODS}

Study population. The WHI-OS is a prospective cohort that enrolled 93676 postmenopausal women aged 50-79 years at 40 clinical centres across the United States between 1993 and 1998 (The Women's Health Initiative Study Group, 1998). We conducted a cross-sectional analysis of baseline data from a nested ovarian and endometrial cancer case-control study within the WHI-OS $(N=1864$ women, $N=510$ cases and 473 controls among never/former MHT users; $N=458$ cases and 423 controls among current MHT users) (Trabert et al, 2015; Brinton et al, 2016). Both dietary information and blood samples were prospectively collected prior to cancer development. Cases and controls were enrolled into the prospective observational study based on the same selection criteria; thus cases and controls were pooled for cross-sectional analysis. These data were obtained an average of $6.9 \pm 3.7$ years prior to endometrial cancer diagnosis and $6.9 \pm 3.8$ years prior to ovarian cancer diagnosis among the cases. Cases were women diagnosed with incident invasive ovarian or endometrial cancer between study enrolment and May 2012, and controls were frequency matched to cases on age at venepuncture $(50-54,55-59,60-64,65-69,70-74,75-79$ years $)$, year at venepuncture (1993-1996, 1997-1998), race/ethnicity (white, black, Hispanic, other/unknown), and time since last MHT use $(\leqslant 1,>1$ year). Controls were alive and cancer-free at the date of diagnosis of their matched case. Eligible women had no history of cancer, besides non-melanoma skin cancer, no history of bilateral oophorectomy or hysterectomy (endometrial controls only), and had adequate prediagnosis serum sample volume available $(1.1 \mathrm{ml})$. Demographic, medical and family history, and lifestyle information was collected via a baseline questionnaire. The study was approved by the institutional review board at the Fred Hutchinson Cancer Research Centre (WHI Clinical Coordinating Centre) and all participating clinical centres. All participants provided written informed consent to participate.

Exposure assessment. Alcohol intake was measured by 122 -item self-administered food frequency questionnaire (FFQ) at the same clinic visit as the fasting venepuncture. The FFQ was previously calibrated against both 24-h dietary recalls and 4-day food records (Patterson et al, 1999). The FFQ asked questions about frequency of intake (never/ $<1$ per month to $\geqslant 6$ servings per day) of specific portion sizes of alcohol ( $12 \mathrm{oz}$ or 1 can beer, $6 \mathrm{oz}$ or 1 medium glass wine, and $1.5 \mathrm{oz}$ or 1 shot liquor). A personal habits questionnaire also inquired about ever alcohol use (12 alcoholic beverages ever- no, yes, missing), and current alcohol use (no, yes, missing). Total alcohol intake (beer, wine and liquor) was calculated, converted into number of standard drinks/week and categorised (non-drinker versus current drinker; drinking categories: $<1,1-<3,3-<7, \geqslant 7$ drinks/week). One serving of beer, wine, or liquor per week contained the same amount of alcohol (approximately $15 \mathrm{~g}$, on average). Alcohol type was also categorised to reflect overall consumption patterns: (1) non-drinker, drinker but not liquor, $>0-1,>1 \mathrm{drink} /$ week for liquor; (2) non-drinker, drinker but not beer, $>0-1,>1$ drink/week for beer; and (3) nondrinker, drinker but not wine, $>0-1,>1-3,>3$ drinks/week for wine).

Laboratory assay. Fifteen fasting serum oestrogens and oestrogen metabolites were quantified using stable isotope dilution liquid chromatography tandem mass spectrometry (LC-MS/MS) (Leidos Biomedical Research, Inc., Frederick, MD, USA) (25). Quantified metabolites included: unconjugated and combined (unconjugated + conjugated) oestrone and oestradiol (parent oestrogens), and 13 oestrogen metabolites (2-hydroxyoestrone, 2-hydroxyoestradiol, 2-hydroxyoestrone-3-methyl ether, 4-hydroxyoestrone, 4-methoxyoestrone, 4-methoxyoestradiol, 16 $\alpha$-hydroxyoestrone, 16-ketoestradiol, 16-epioestriol, 17-epioestriol, as well as unconjugated and combined oestriol, 2-methoxyoestrone, and 2-methoxyoestradiol). Conjugated concentrations of oestrone, oestradiol, oestriol, 2-methoxyoestrone, and 2-methoxyoestradiol were calculated by subtracting unconjugated from total combined concentration. Assay reliability was assessed using masked technical replicates analysed across batches. Coefficients of variation (CV) were $<6 \%$ for all analytes; median (range) intraclass correlation coefficients (ICC) across all analytes was 0.98 (0.93-0.996) (Trabert et al, 2015; Brinton et al, 2016).

\section{Statistical analysis}

Primary analysis. Since the serum samples for the nested casecontrol study of ovarian and endometrial cancer were collected prior to diagnosis, we included both cases and controls $(N=1864)$ in this cross-sectional analysis. Geometric means (GM, pmoll ${ }^{-1}$ ) of oestrogen/oestrogen metabolite concentrations were calculated by (1) overall alcohol exposure category and (2) alcohol type category (liquor, beer, wine) using inverse probability weighted linear regression. Analyses were re-weighted by inverse probability sampling weights to account for case-control selection criteria and 
to represent the WHI-OS cohort (Li and Gail, 2012). Sampling weights were the inverse of sampling fractions for cases (one) and controls (adjusted depending on strata and matching factors). Serum concentrations of oestrogen/oestrogen metabolites were evaluated individually, and in unconjugated and combined forms for select analytes. Analytes were log-transformed to account for non-normality. All analyses were multivariable and adjusted for a priori-defined potential confounders (Key et al, 2003; Setiawan et al, 2006; Chan et al, 2007; Kotsopoulos et al, 2009; Friedenreich et al, 2010; Brand et al, 2011; Sisti et al, 2015): venepuncture age $(<60,60-<70, \geqslant 70$ years), venepuncture year (1993-1996, 1997-1998), race (white, non-white), smoking status (never, past, current), body mass index (BMI) $\left(\mathrm{kg} \mathrm{m}^{-2}\right.$, continuous), years since menopause $(<10$ years, $10-<20$ years, $\geqslant 20)$, oral contraceptive use duration $(0,<5, \geqslant 5$ years), caffeine intake (quartiles), sedentary/sitting time $(<5,5-<10, \geqslant 10 \mathrm{~h}$ per day), and metabolic equivalent (MET) hours of physical activity/week (none, $>0-<7.5,7.5-15$ (National recommendations), $\geqslant 15$ ). We conducted Wald tests for trend among alcohol drinkers using the median values of the alcohol categories (servings/week). We ran restricted cubic spline regression on alcohol associations with unconjugated and conjugated parent oestrogens, creating 5-knot splines at the 5th, 25th, 50th, 75th, and 95th percentiles of number of alcohol servings/week (Durrleman and Simon, 1989). The main analyses were stratified by never/former MHT users versus current users given that oestrogen/oestrogen metabolite concentrations vary by MHT use.

Sensitivity analyses. Stratification and Effect Modification: In sensitivity analyses, we excluded: (1) cancer cases, (2) women with diabetes, (3) oestrogen metabolite outliers, (4) alcohol intake outliers. We tested for effect modification with the inclusion of an alcohol-modifier interaction term in the main analyses. Outliers were identified using an extreme studentised deviate many-outlier procedure (Rosner, 1983).

Confounding: We evaluated the role of MHT formulation (unopposed oestrogen use, both oestrogen and progesterone use, or a combination of one of these formulations with past use of the other formulation) by further adjusting analyses of total alcohol intake and oestrogen/oestrogen metabolite concentrations for MHT formulation among current MHT users. MHT dosage did not vary substantially among MHT users. We also further adjusted select metabolite findings for parent oestrogens to assess whether their associations with alcohol were independent. We additionally controlled alcohol type for total alcohol consumption. We also checked for residual confounding by smoking by adjusting for cigarette pack years.

Metabolite ratio: Finally, we evaluated associations between overall alcohol intake and oestrogen/oestrogen metabolite ratios, including: ratio of parent to total oestrogens; ratio of unconjugated to combined oestrone, oestradiol, oestriol, 2-methoxyoestrone, and 2-methoxyoestradiol; ratio of 2-hydroxyoestrone to 4-hydroxyoestrone; ratio of 2-hydroxyoestrone to 16-alphahydroxyoestrone, and ratio of 4-hydroxyoestrone to 16-alphahydroxyoestrone. We calculated $P$-value for trends of increasing/decreasing metabolite ratios across alcohol drinking categories.

All $P$-values were two-sided and considered statistically significant if less than 0.05 . For descriptive purposes, we also calculated the false discovery rate (FDR), and set a threshold of less than 0.05 as a second, more stringent, threshold of statistical significance (Benjamini-Hochberg procedure) (Benjamini and Hochberg, 1995; Ganna et al, 2014). All analyses were conducted with appropriate sampling weights using SAS Survey Procedures (PROC SURVEYREG, SAS version 9.3, SAS Institute, Cary, NC, USA).

\section{RESULTS}

Study population. In this cross-sectional analysis among 1864 women participating in the WHI-OS, never/former MHT users were aged $64 \pm 7$ years and current MHT users were aged $63 \pm 7$ years at their baseline visit, on average (Table 1). Most women were Caucasian, non-smokers that met national physical activity guidelines ( $\geqslant 7.5$ MET hours per week), had an average BMI in the overweight range $\left(25-<30 \mathrm{~kg} \mathrm{~m}^{-2}\right)$, and had been in menopause for $>10$ years. Thirty-nine percent of never/former MHT users and $55 \%$ of current MHT users had a history of oral contraceptive use.

Alcohol intake. The median (interdecile range) servings/week of alcohol was $0.4(0,8.3)$ among never/former MHT users and $0.7(0$, 8.7) among current MHT users. Among drinkers, median (interdecile range) servings/week alcohol was $2.6(0.2,16.0)$ among never/former MHT users and $2.7(0.4,10.9)$ among current MHT users. Twenty-nine percent of never/former MHT users reported not currently being alcohol drinkers $(N=280)$, compared with $22 \%$ of current MHT users $(N=190)$. Median (interdecile range) standard drinks/week of alcohol types were: (1) beer $0.42(0.21$, $7.00)$, liquor $1.00(0.21,7.88)$, wine $1.00(0.21,7.00)$ among never/ former MHT users; (2) beer $0.42(0.21,2.72)$, liquor 0.49 (0.21, $7.00)$, wine $1.36(0.21,7.88)$ among current MHT users.

Among never/former MHT users, the highest category of drinkers had higher circulating oestrogens and 2-, 4-, and 16pathway metabolite concentrations compared with no current alcohol intake, although associations were not statistically significant (Table 2). Among MHT users who were drinkers, increasing alcohol intake (GM for $7+$ compared with $<1$ drink per week) was associated with higher concentrations of unconjugated oestrone $(26 \%$ increase, $p$-trend $=0.01)$, unconjugated and conjugated oestradiol (26 and $29 \%$ increases, respectively, $p$-trend $=0.04)$, unconjugated 2-methoxyoestrone $(37 \%$ increase, $p$-trend $=0.01)$, and unconjugated 2-methoxyoestradiol $(17 \%$ increase, $p$-trend $=0.04$ ) (Table 3). However, these trends did not meet FDR $<0.05$. The trends for increasing unconjugated oestrone $(P=0.02)$ and oestradiol $(P=0.03)$ with increasing servings of alcohol/week among MHT users were linear based on cubic spline regression analysis (results not shown). Results were not materially changed with finer adjustment for cigarette pack years (analyte concentrations changed 1-12\%), although nominal statistical significance was retained only for increasing trends of unconjugated oestrone, 2-methoxyestrone, and 2-methoxyestradiol among current MHT users.

Among never/former MHT users, liquor consumption was associated with $19-32 \%$ increases in GM of parent oestrogen and oestrogen metabolite concentration for highest level compared with no intake of that alcohol type ( $p$-trend across current liquor drinkers $=0.001$ to 0.04 ), including: combined and conjugated oestrone, unconjugated oestradiol, 2-hydroxyoestrone, and 2-hydroxyoestradiol, combined and conjugated 2-methoxyoestrone, unconjugated 2-methoxyoestradiol, 3-methyl ether-hydroxyoestrone, 4-hydroxyoestrone, 4-methoxyoestrone, 16-alpha-hydroxyoestrone, all three measures of oestriol, 16-ketoestradiol, 16-epioestriol, and 17 -epioestriol. FDR was $<0.05$ for all associations (Table 4; see Supplementary Table 1 for current MHT users). These associations were not retained after further adjustment for parent oestrogens. Consumption of beer and wine were not associated with oestrogen/oestrogen metabolites. Findings were not materially altered with further adjustment for total alcohol intake (data not shown).

Since we observed alcohol associations primarily with the unconjugated component of oestrogen/oestrogen metabolites, we compared the proportion of unconjugated to combined 
Table 1. Characteristics of study participants in the Women's Health Initiative (WHI) Observational Study (OS) by alcohol category, stratified by menopausal hormone therapy (MHT) use

\begin{tabular}{|c|c|c|c|c|c|c|c|c|c|c|c|c|c|c|c|c|c|c|}
\hline \multirow[b]{3}{*}{ Characteristic } & \multicolumn{9}{|c|}{ Never/former MHT users $(N=983)^{a}$} & \multicolumn{9}{|c|}{ Current MHT users $(N=881)$} \\
\hline & \multicolumn{3}{|c|}{ Total } & \multicolumn{3}{|c|}{ Non-drinker } & \multicolumn{3}{|c|}{ Current drinker } & \multicolumn{3}{|c|}{ Total } & \multicolumn{3}{|c|}{ Non-drinker } & \multicolumn{3}{|c|}{ Current drinker } \\
\hline & $N$ & Wt $N$ & $(\%)^{b}$ & $N$ & Wt $N$ & $(\%)^{b}$ & $N$ & Wt N & $(\%)^{b}$ & $N$ & Wt $N$ & $(\%)^{b}$ & $N$ & Wt $N$ & $(\%)^{b}$ & $N$ & Wt N & $(\%)^{b}$ \\
\hline$<60$ & 259 & 8029 & (26) & 106 & 3000 & (10) & 153 & 5029 & (16) & 297 & $\mid 10792$ & (43) & 84 & 3471 & (14) & 213 & 7321 & (29) \\
\hline $60-69$ & 433 & 13635 & (44) & 170 & 5079 & (16) & 263 & 8556 & (28) & 397 & 10689 & (42) & 129 & 3765 & (15) & 268 & 6924 & (27) \\
\hline 70-79 & 961 & 31002 & (30) & 114 & 3802 & (12) & 155 & 5536 & (18) & 187 & 3840 & (15) & 65 & 1607 & (6) & 122 & 2233 & (9) \\
\hline 1997-1998 & 373 & 12014 & (39) & 154 & 4733 & (15) & 219 & 7280 & (23) & 335 & 9666 & (38) & 114 & 3370 & (13) & 221 & 6296 & $(25)$ \\
\hline \multicolumn{19}{|l|}{ Race } \\
\hline White & 844 & 27738 & (89) & 310 & 9684 & (31) & 534 & \begin{tabular}{|l|}
18054 \\
\end{tabular} & (58) & 827 & 23616 & (93) & 244 & 7736 & (31) & 583 & 15880 & (63) \\
\hline Non-white & 117 & 3264 & (11) & 80 & 2197 & (7) & 37 & 1067 & (3) & 54 & 1705 & $(7)$ & 34 & 1108 & (4) & 20 & 597.5 & (2) \\
\hline \multicolumn{19}{|l|}{ Smoking status } \\
\hline$<25$ & 349 & 13671 & (44) & 112 & 4214 & (14) & 237 & 9457 & (31) & 447 & 11554 & (46) & 116 & 3317 & (13) & 331 & 8236 & (33) \\
\hline $25-<30$ & 295 & 9352 & (30) & 125 & 4180 & (14) & 170 & 5172 & (17) & 258 & 7903 & (31) & 85 & 2788 & (11) & 173 & 5115 & (20) \\
\hline $30+$ & 315 & 7894 & (26) & 151 & 3401 & (11) & 164 & 4493 & (15) & 175 & 5810 & (23) & 77 & 2738 & (11) & 98 & 3071 & $(12)$ \\
\hline \multicolumn{19}{|c|}{ Years since menopause } \\
\hline$<10$ & 299 & 9267 & (31) & 105 & 3144 & (11) & 194 & 6123 & (21) & 351 & 11154 & (44) & 95 & 3204 & (13) & 256 & 7950 & (31) \\
\hline $10-<20$ & 361 & 11390 & (39) & 146 & 3953 & (13) & 215 & 7437 & (25) & 331 & 8838 & (35) & 111 & 3472 & (14) & 220 & 5367 & (21) \\
\hline $20+$ & 252 & 8888 & (30) & 114 & 3924 & (13) & 138 & 4964 & (17) & 199 & 5328 & (21) & 72 & 2167 & (9) & 127 & 3161 & (12) \\
\hline \multicolumn{19}{|c|}{ Duration of oral contraceptive use (years) } \\
\hline Never & 618 & 18930 & (61) & 255 & 7722 & (25) & 363 & \begin{tabular}{|l|l|l|l|l}
11208 \\
\end{tabular} & (36) & 453 & 11445 & (45) & 155 & 4158 & (16) & 298 & 7287 & $(29)$ \\
\hline$<5$ & 182 & 6476 & (21) & 79 & 2367 & (8) & 103 & 4109 & (13) & 212 & 6085 & (24) & 59 & 1833 & (7) & 153 & 4252 & (17) \\
\hline $5+$ & 160 & 5518 & (18) & 55 & 1715 & (6) & 105 & 3804 & (12) & 216 & 7791 & (31) & 64 & 2853 & (11) & 152 & 4938 & $(20)$ \\
\hline \multicolumn{19}{|c|}{ Caffeine intake quartile (median mg per day) } \\
\hline MET hours $p$ & veek & & & & & & & & & & & & & & & & & \\
\hline None & 206 & 6139 & (20) & 99 & 2814 & (9) & 107 & 3325 & (11) & 145 & 4642 & (18) & 67 & 2188 & (9) & 78 & 2454 & (10) \\
\hline$>0-<7.5$ & 216 & 6724 & (22) & 96 & 2724 & (9) & 120 & 4000 & (13) & 216 & 7180 & (28) & 69 & 2319 & (9) & 147 & 4861 & (19) \\
\hline 7.5-15 & 224 & 7586 & (25) & 89 & 3111 & (10) & 135 & 4475 & (15) & 196 & 4671 & (18) & 73 & 2174 & (9) & 123 & 2497 & (10) \\
\hline$\geqslant 15$ & 303 & 10407 & (34) & 101 & 3171 & (10) & 202 & 7236 & (23) & 319 & 8771 & (35) & 68 & 2109 & (8) & 251 & 6661 & (26) \\
\hline obreviation & & $t=$ & olic & aler & & & & & & heted & & & & & & & & \\
\hline & & & & & & & & & & & & & & & & & & \\
\hline
\end{tabular}

concentration for these hormones across drinking categories (Supplementary Table 2). Trends for decreasing ratio of unconjugated to combined oestrone ( $18 \%$ versus $20 \%$ for $7+$ compared with $<1$ drink per week) and 2-methoxyoestradiol (16\% versus $18 \%$ for $7+$ compared with $<1$ drink per week) were evident among never/former MHT users, while a trend for increasing unconjugated to combined 2-methoxyoestrone ( 40 vs 33\% for $7+$ compared with $<1$ drink per week) was evident for increasing categories of alcohol intake among current MHT users. For liquor, highest category compared with no liquor consumption was inversely associated with ratio of unconjugated to combined oestrone (17 vs $21 \%$ for $>1$ compared with no liquor intake). These trends did not meet FDR $<0.05$.

We further excluded cases $(N=510$ never/former MHT users; $N=458$ current MHT users; Supplementary Table 3) and women with diabetes $(N=42$ never/former MHT users; $N=28$ current
MHT users; results not shown) from the overall alcohol and liquor analyses. There were no differences in the association between overall alcohol consumption and oestrogen/oestrogen metabolites between cases and controls, or between women with and without diabetes. There was no evidence for alcohol-case status effect modification (all $P$-values for interaction $>0.05$ ). Similarly, excluding cases and women with diabetes did not alter the patterns of association for liquor consumption. When we removed oestrogen metabolite outliers or alcohol outliers (results not shown), unconjugated oestrone and 2-methoxyoestrone remained nominally associated with overall alcohol consumption among MHT users. When we additionally adjusted for MHT formulation (Supplementary Table 4), we observed similar trends among MHT users for the main effects of alcohol consumption on oestrogen and 2-hydroxylation pathway catechol oestrogen metabolites. 
Table 2. Geometric means (pmoll ${ }^{-1}$ ) and $95 \%$ confidence intervals $(\mathrm{Cl}$ ) of serum oestrogens and oestrogen metabolites by total alcohol consumption category among postmenopausal women not using menopausal hormone therapy in the Women's Health Initiative (WHI) Observational Study (OS)

\begin{tabular}{|c|c|c|c|c|c|c|c|c|c|}
\hline $\begin{array}{l}\text { Alcoholic drinks } \\
\text { per week }\end{array}$ & Non-drinker & $\begin{array}{l}\text { Current } \\
\text { drinker }\end{array}$ & & $<1$ & 1 to $<3$ & 3 to $<7$ & $7+$ & & \\
\hline Median & 0 & 2.6 & & 0.4 & 1.8 & 4 & 11.5 & & \\
\hline$N$ & 398 & 565 & & 198 & 138 & 101 & 148 & & \\
\hline \multirow[t]{2}{*}{ Weighted $N^{a}$} & 11881 & 19121 & & 6560 & 4195 & 3322 & 5044 & & \\
\hline & \multicolumn{2}{|c|}{$\begin{array}{c}\text { Geometric means } \\
(95 \% \mathrm{Cl})^{\mathbf{b}}\end{array}$} & $\begin{array}{c}P- \\
\text { diffe }\end{array}$ & \multicolumn{4}{|c|}{$\begin{array}{c}\text { Geometric means } \\
(95 \% \mathrm{Cl})^{\mathbf{b}}\end{array}$} & $\% \Delta^{d}$ & $\begin{array}{c}p- \\
\text { trend }^{\mathbf{e}}\end{array}$ \\
\hline Oestrone & $313.8(266.1,370.0)$ & $332.6(278.3,397.6)$ & 0.50 & $314.3(255.2,387.1)$ & $290.7(223.2,378.6)$ & $403.3(301.2,540.0)$ & $340.4(268.7,431.3)$ & $8 \%$ & 0.66 \\
\hline Conjugated & $245.2(204.4,294.1)$ & $266.8(218.9,325.3)$ & 0.37 & $249.2(197.6,314.3)$ & $229.8(171.1,308.6)$ & $320.5(231.6,443.7)$ & $279.3(216.0,361.1)$ & $12 \%$ & 0.50 \\
\hline Unconjugated & $60.6(53.7,68.5)$ & $58.9(51.8,66.9)$ & 0.66 & $58.9(50.5,68.6)$ & $52.9(44.3,63.1)$ & $73.9(60.0,90.9)$ & $55.5(46.6,66.3)$ & $-6 \%$ & 0.51 \\
\hline Oestradiol & $60.0(50.3,71.6)$ & $57.7(47.6,70.0)$ & 0.67 & $53.8(43.1,67.3)$ & $53.6(42.4,67.9)$ & $72.0(52.2,99.3)$ & $57.3(44.6,73.7)$ & $6 \%$ & 0.82 \\
\hline Conjugated & $38.6(32.0,46.7)$ & $39.7(32.1,49.1)$ & 0.77 & $36.5(28.4,46.9)$ & $39.5(30.5,51.1)$ & $47.6(33.9,66.8)$ & $39.3(29.7,52.2)$ & $8 \%$ & 0.88 \\
\hline Unconjugated & $15.9(13.1,19.3)$ & $13.5(11.2,16.1)$ & 0.09 & $13.2(10.6,16.4)$ & $10.7(8.4,13.7)$ & $17.6(12.8,24.3)$ & $13.4(10.8,16.7)$ & $2 \%$ & 0.67 \\
\hline 2-Hydroxyoestrone & $68.7(59.4,79.5)$ & $68.1(58.2,79.8)$ & 0.91 & $65.8(54.6,79.2)$ & $59(47.3,73.7)$ & $80.4(61.2,105.6)$ & $69.6(57.7,84.1)$ & $6 \%$ & 0.52 \\
\hline 2-Hydroxyoestradiol & $16.9(14.6,19.5)$ & $16.9(14.6,19.7)$ & 0.97 & $16.5(13.8,19.9)$ & $14.6(11.7,18.1)$ & $19.7(15.2,25.5)$ & $17.3(14.4,20.9)$ & $5 \%$ & 0.52 \\
\hline 2-Methoxyoestrone & $43.5(38.6,48.9)$ & $43.3(38.2,49.1)$ & 0.95 & $42.1(35.9,49.4)$ & $38.2(31.9,45.8)$ & $49.0(39.2,61.3)$ & $44.4(37.6,52.6)$ & $6 \%$ & 0.38 \\
\hline Conjugated & $31.9(28.0,36.3)$ & $31.9(27.9,36.5)$ & 0.98 & $30.8(25.8,36.8)$ & $27.8(22.9,33.7)$ & $35.0(27.6,44.5)$ & $33.7(28.2,40.2)$ & $9 \%$ & 0.23 \\
\hline Unconjugated & $10.3(9.0,11.9)$ & $10.0(8.6,11.6)$ & 0.65 & $9.9(8.2,12.1)$ & $9.4(7.5,11.7)$ & $12.1(9.3,15.7)$ & $9.5(7.7,11.6)$ & $-5 \%$ & 0.63 \\
\hline 2-Methoxyoestradiol & $13.9(12.0,16.2)$ & $14.1(11.9,16.8)$ & 0.81 & $13.8(11.5,16.6)$ & $12.1(9.7,15.2)$ & $17(12.7,22.8)$ & $14.3(11.5,17.7)$ & $4 \%$ & 0.56 \\
\hline Conjugated & $11.2(9.5,13.3)$ & $11.5(9.5,13.8)$ & 0.75 & $11.1(9.1,13.6)$ & $9.3(7.2,12.2)$ & $14.0(10.3,19.2)$ & $11.9(9.5,14.9)$ & $7 \%$ & 0.32 \\
\hline Unconjugated & $2.2(1.9,2.5)$ & $2.1(1.8,2.4)$ & 0.56 & $2.1(1.7,2.5)$ & $2.0(1.7,2.5)$ & $2.3(1.8,3.0)$ & $2.0(1.7,2.4)$ & $-2 \%$ & 0.48 \\
\hline $\begin{array}{l}\text { 3-Methyl ether- } \\
\text { hydroxyoestrone }\end{array}$ & $7.7(6.8,8.7)$ & $7.8(6.8,9)$ & 0.76 & $7.7(6.5,9.0)$ & $7.0(5.8,8.4)$ & $8.2(6.4,10.4)$ & $8.2(6.9,9.8)$ & $7 \%$ & 0.51 \\
\hline 4-Hydroxyoestrone & $8.4(7.3,9.7)$ & $8.5(7.3,10.0)$ & 0.85 & $8.3(6.9,10.0)$ & $7.4(5.9,9.2)$ & $9.9(7.5,13.1)$ & $8.6(7.1,10.4)$ & $4 \%$ & 0.61 \\
\hline 4-Methoxyoestrone & $4.6(4.1,5.2)$ & $4.5(3.9,5.1)$ & 0.71 & $4.6(3.9,5.4)$ & $3.9(3.2,4.6)$ & $4.9(3.8,6.2)$ & $4.6(3.9,5.4)$ & $-1 \%$ & 0.57 \\
\hline 4-Methoxyoestradiol & $2.0(1.7,2.3)$ & $2.0(1.7,2.4)$ & 0.65 & $2.0(1.7,2.5)$ & $1.8(1.5,2.3)$ & $2.4(1.8,3.2)$ & $2.0(1.6,2.4)$ & $-2 \%$ & 0.95 \\
\hline $\begin{array}{l}\text { 16- } \\
\text { Alphahydroxyoestrone }\end{array}$ & $34.3(29.5,40.0)$ & $34.0(28.7,40.2)$ & 0.90 & $32.6(26.8,39.6)$ & $29.5(23.3,37.4)$ & $40.2(29.7,54.4)$ & $34.9(28.7,42.5)$ & $7 \%$ & 0.51 \\
\hline Oestriol & $147.1(127.1,170.2)$ & $144.5(122.5,170.5)$ & 0.82 & $142.0(116.6,172.9)$ & $126.7(99.4,161.6)$ & $171.0(127.2,229.9)$ & $144.3(118.5,175.7)$ & $2 \%$ & 0.88 \\
\hline Conjugated & $115.6(97.8,136.7)$ & $114.3(95,137.6)$ & 0.90 & $111.9(89.5,139.8)$ & $101.4(77.6,132.6)$ & $137.3(99.8,188.9)$ & $113.1(90.4,141.5)$ & $1 \%$ & 0.99 \\
\hline Unconjugated & $27.8(24.7,31.3)$ & $26.0(22.8,29.8)$ & 0.38 & $25.7(21.8,30.3)$ & $21.8(17.6,27)$ & $29.5(22.7,38.4)$ & $27.1(23,31.8)$ & $5 \%$ & 0.25 \\
\hline 16-Ketooestradiol & $36.9(31.6,43.1)$ & $37.3(31.5,44.3)$ & 0.88 & $36.3(29.7,44.5)$ & $32.2(25.1,41.3)$ & $43.0(31.7,58.5)$ & $38.4(31.3,47.1)$ & $6 \%$ & 0.64 \\
\hline 16-Epioestriol & $15.8(13.7,18.1)$ & $15.5(13.3,18.1)$ & 0.80 & $14.2(11.9,17.1)$ & $13.8(11.1,17.1)$ & $18.5(14.3,24.1)$ & $16.2(13.5,19.5)$ & $14 \%$ & 0.26 \\
\hline 17-Epioestriol & $12.4(10.9,14.2)$ & $12.7(11,14.6)$ & 0.76 & $11.9(10.0,14.2)$ & $11.1(9.1,13.6)$ & $14.6(11.6,18.4)$ & $13.4(11.1,16.1)$ & $12 \%$ & 0.28 \\
\hline \multicolumn{10}{|c|}{ 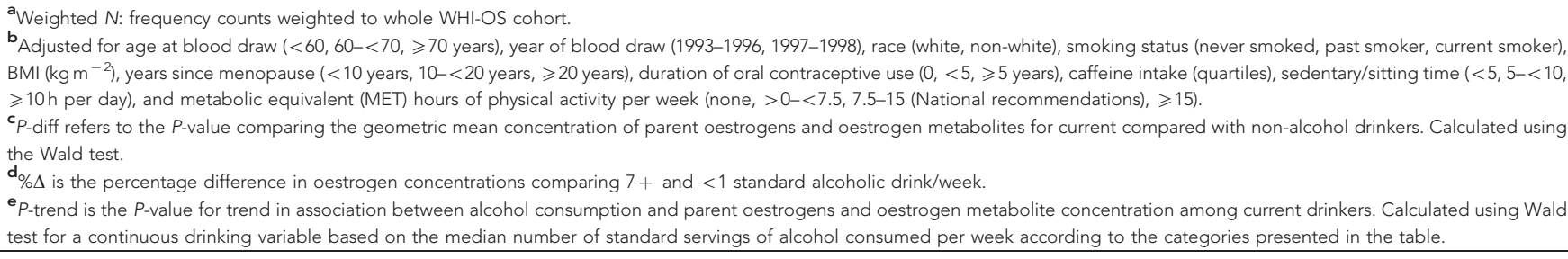 } \\
\hline
\end{tabular}

\section{DISCUSSION}

In this population of 1864 postmenopausal women from a nested case-control study within the WHI-OS cohort, we observed nominal associations for increasing concentrations of parent oestrogens with increasing alcohol intake among both never/former and current MHT users, consistent with prior studies. We also observed trends of increased 2-hydroxylation pathway oestrogen metabolites with increasing alcohol intake, although trends did not meet the FDR threshold. Liquor was associated with increased concentrations of circulating parent oestrogens and 2-, 4-, and 16-pathway oestrogen metabolites among never/former MHT users across frequency of use categories; oestrogen metabolite associations with liquor intake did not remain with further adjustment for parent oestrogens.
Prior randomised dietary interventions and prospective studies have supported that alcohol intake is associated with postmenopausal hormone levels. The Women's Alcohol study, a placebo controlled 8-week crossover feeding study among 51 healthy postmenopausal women not using MHT, showed that 15-30 g per day alcohol intake increased serum oestrone sulphate by $7.5-10.7 \%$ and DHEAS by 5.1-7.5\% (Dorgan et al, 2001). In a pooled metaanalysis of 13 prospective studies $(N=6291)$ from across the globe, sex hormone concentrations were $\sim 10-25 \%$ higher among postmenopausal women consuming $\geqslant 20 \mathrm{~g}$ alcohol per day ( $\sim 20 \%$ women) compared with non-drinkers, including oestradiol, oestrone, androstenedione, DHEAS, and testosterone; SHBG concentrations were reduced by $10 \%$ (Key et al, 2011). Fifteen to $30 \mathrm{~g}$ alcohol per day approximates one to two standard drinks/day, in contrast to median intakes of $\sim 0.4$ standard drinks/day among drinkers in our population. The effect sizes we observed in our analysis for parent oestrogens were consistent with these prior 
Table 3. Geometric means (pmoll ${ }^{-1}$ ) and $95 \%$ confidence intervals $(\mathrm{CI})$ of serum oestrogens and oestrogen metabolites by total alcohol consumption category among postmenopausal women currently using menopausal hormone therapy in the Women's Health Initiative (WHI) Observational Study (OS)

\begin{tabular}{|c|c|c|c|c|c|c|c|c|c|}
\hline $\begin{array}{l}\text { Alcoholic drinks } \\
\text { per week }\end{array}$ & Nondrinker & $\begin{array}{l}\text { Current } \\
\text { drinker }\end{array}$ & & $<1$ & 1 to $<3$ & 3 to $<7$ & $7+$ & & \\
\hline Median & 0 & 2.7 & & 0.4 & 1.9 & 4.5 & 10.5 & & \\
\hline N & 278 & 603 & & 199 & 136 & 125 & 142 & & \\
\hline \multirow[t]{2}{*}{ Weighted $N^{a}$} & 8843 & 16478 & & 5310 & 3639 & 3694 & 3779 & & \\
\hline & \multicolumn{2}{|c|}{ Geometric means $(95 \% \mathrm{CI})^{\mathbf{b}}$} & $P$-diff ${ }^{c}$ & \multicolumn{4}{|c|}{ Geometric means $(95 \% \mathrm{CI})^{\mathbf{b}}$} & $\% \Delta^{d}$ & $p$-trend ${ }^{\mathbf{e}}$ \\
\hline Oestrone & $2835.3(2147.4,3743.5)$ & $3468.8(2606.5,4616.4)$ & 0.10 & $3411.3(2476.0,4700.0)$ & $3171.6(2051.0,4904.4)$ & $3386.1(2320.6,4940.9)$ & $4013.1(2808.6,5734.1)$ & $18 \%$ & 0.10 \\
\hline Conjugated & $2601.9(1955.2,3462.6)$ & $3167.2(2361.9,4247.1)$ & 0.12 & $3196.5(2301.7,4439.3)$ & $2806.4(1785.0,4412.4)$ & $3091.4(2094.2,4563.5)$ & $3614.9(2499.4,5228.2)$ & $13 \%$ & 0.15 \\
\hline Unconjugated & $205.5(167.9,251.5)$ & $221.3(178.6,274.2)$ & 0.45 & $218.8(171.0,280.0)$ & $174.4(127.0,239.6)$ & $231.6(170.2,315.3)$ & $276.2(209.9,363.3)$ & $26 \%$ & $0.01^{f}$ \\
\hline Oestradiol & $403.7(306.3,532.2)$ & $438.5(333.7,576.2)$ & 0.48 & $393.9(286.2,542.3)$ & $435.5(289.3,655.7)$ & $445.8(312.0,637.0)$ & $525.4(376.5,733.2)$ & $33 \%$ & $0.02^{f}$ \\
\hline Conjugated & $342.8(251.1,468.1)$ & $360.4(267.5,485.4)$ & 0.70 & $338.1(239.8,476.8)$ & $344.7(217.4,546.3)$ & $350.0(237.7,515.4)$ & $436.8(302.8,630.0)$ & $29 \%$ & $0.04^{f}$ \\
\hline Unconjugated & $40.4(32.0,51.0)$ & $45.8(36.2,57.8)$ & 0.21 & $41.7(31.3,55.7)$ & $41.2(30.1,56.5)$ & $52.6(36.8,75.1)$ & $52.6(38.8,71.3)$ & $26 \%$ & $0.04^{f}$ \\
\hline 2-Hydroxyoestrone & $408.2(329.3,506.1)$ & $441.9(356.4,547.9)$ & 0.42 & $447.5(345.6,579.4)$ & $409.0(299.1,559.3)$ & $437.9(321.8,595.8)$ & $472.2(358.2,622.4)$ & $6 \%$ & 0.25 \\
\hline 2-Hydroxyoestradiol & $100.0(82.1,121.8)$ & $101.3(82.5,124.4)$ & 0.89 & $105.6(82.4,135.4)$ & $89.1(66.2,119.9)$ & $99.7(73.9,134.7)$ & $109.1(83.5,142.4)$ & $3 \%$ & 0.25 \\
\hline 2-Methoxyoestrone & $250.8(209.8,299.9)$ & $248.4(206.2,299.1)$ & 0.92 & $248.6(195.3,316.5)$ & $202.1(152.8,267.4)$ & $270.2(208.2,350.6)$ & $281.7(218.6,363.1)$ & $13 \%$ & 0.05 \\
\hline Conjugated & $155.4(124.4,194.0)$ & $149.3(120.3,185.2)$ & 0.69 & $157.7(121.6,204.6)$ & $126.1(92.3,172.1)$ & $153.3(115.2,204.1)$ & $156.5(116.7,209.9)$ & $-1 \%$ & 0.46 \\
\hline Unconjugated & $68.9(53.1,89.4)$ & $72.0(55.8,92.9)$ & 0.73 & $67.4(48.7,93.3)$ & $54.6(38.4,77.5)$ & $84.4(58.6,121.6)$ & $92.4(65.8,129.6)$ & $37 \%$ & $0.01^{f}$ \\
\hline 2-Methoxyoestradiol & $81.5(63.2,105.2)$ & $80.3(63.1,102.2)$ & 0.88 & $77.6(58.8,102.4)$ & $69.5(49.4,97.9)$ & $84.0(61.6,114.6)$ & $94.6(70.6,126.8)$ & $22 \%$ & $0.02^{f}$ \\
\hline Conjugated & $70.8(53.7,93.3)$ & $66.4(51.1,86.1)$ & 0.54 & $66.8(49.6,90.1)$ & $56.0(38.5,81.5)$ & $67.2(48.2,93.6)$ & $77.2(56.2,106.1)$ & $16 \%$ & 0.06 \\
\hline Unconjugated & $8.0(6.6,9.7)$ & $8.9(7.5,10.7)$ & 0.23 & $8.7(6.9,11.1)$ & $7.7(5.9,10.2)$ & $9.5(7.1,12.7)$ & $10.2(8.0,13.0)$ & $17 \%$ & $0.04^{f}$ \\
\hline $\begin{array}{l}\text { 3-Methyl ether- } \\
\text { hydroxyoestrone }\end{array}$ & $39.4(32.1,48.4)$ & $39.4(32.1,48.3)$ & 0.99 & $40.8(32.1,51.9)$ & $30.5(23.0,40.6)$ & $43.5(33.4,56.7)$ & $43.7(33.7,56.6)$ & $7 \%$ & 0.09 \\
\hline 4-Hydroxyoestrone & $54.1(43.6,67)$ & $58.9(47.3,73.4)$ & 0.39 & $59.9(46.1,77.8)$ & $54.4(39.7,74.7)$ & $59.2(43.4,80.7)$ & $61.9(46.8,81.9)$ & $3 \%$ & 0.33 \\
\hline 4-Methoxyoestrone & $24.9(20.6,30.1)$ & $25.7(21.4,30.9)$ & 0.75 & $25.9(20.4,32.9)$ & $21.2(16.3,27.7)$ & $28.5(22.1,36.6)$ & $27.7(21.9,35.1)$ & $7 \%$ & 0.16 \\
\hline 4-Methoxyoestradiol & $10.6(8.0,13.9)$ & $10.7(8.2,13.9)$ & 0.91 & $10.6(7.8,14.4)$ & $9.0(6.3,13.0)$ & $11.1(7.9,15.6)$ & $12.5(9.2,17.0)$ & $18 \%$ & 0.05 \\
\hline 16-Alphahydroxyoestrone & $216.0(172.0,271.3)$ & $235.0(187.9,294.0)$ & 0.40 & $239.9(183.9,312.9)$ & $213.8(155.4,293.9)$ & $237.8(172.8,327.3)$ & $247.1(185.1,329.7)$ & $3 \%$ & 0.30 \\
\hline Oestriol & $983.7(770.7,1255.5)$ & $1134.9(886.5,1453)$ & 0.19 & $1105.7(833.1,1467.6)$ & $1110.2(776.9,1586.4)$ & $1210.8(850.2,1724.4)$ & $1140.0(830.3,1565.1)$ & $3 \%$ & 0.47 \\
\hline Conjugated & $849.8(659.8,1094.5)$ & $975.5(754.7,1261.1)$ & 0.23 & $967.7(719.1,1302.3)$ & $946.7(654.7,1369.0)$ & $1049.9(724.8,1520.9)$ & $949.5(675.6,1334.5)$ & $-2 \%$ & 0.69 \\
\hline Unconjugated & $117.9(96.3,144.4)$ & $122.3(98.5,151.9)$ & 0.68 & $129.3(101.6,164.6)$ & $95.1(69.9,129.3)$ & $133.0(98.8,179.1)$ & $132.0(101.4,171.8)$ & $2 \%$ & 0.20 \\
\hline 16-Ketooestradiol & $245.2(195.0,308.5)$ & $275.3(219.9,344.8)$ & 0.27 & $277.7(212.4,363.0)$ & $251.2(180.7,349.2)$ & $286.1(205.6,398.0)$ & $287.0(213.4,385.9)$ & $3 \%$ & 0.34 \\
\hline 16-Epioestriol & $77.0(62.6,94.8)$ & $79.6(64.4,98.3)$ & 0.74 & $83.8(65.2,107.7)$ & $68.6(50.5,93.3)$ & $84.6(64.1,111.7)$ & $79.6(60.2,105.3)$ & $-5 \%$ & 0.56 \\
\hline 17-Epioestriol & $54.2(43.8,67.0)$ & $54.8(44.0,68.3)$ & 0.91 & $56.4(43.6,73.0)$ & $48.4(35.3,66.5)$ & $59.6(44.6,79.7)$ & $54.4(40.3,73.5)$ & $-4 \%$ & 0.58 \\
\hline \multicolumn{10}{|c|}{ 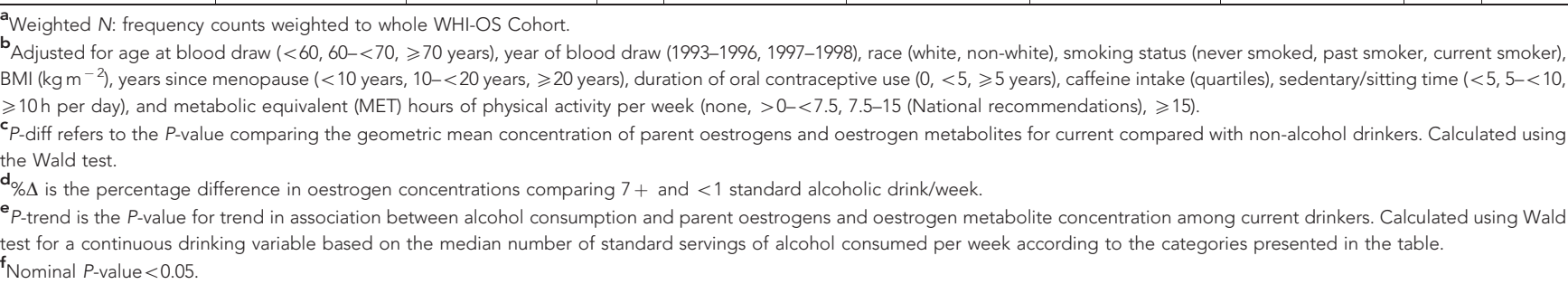 } \\
\hline
\end{tabular}

studies, but did not reach statistical significance, possibly due to sample size.

Few prior studies have evaluated associations of alcohol consumption with circulating sex steroid hormone metabolites, which may provide insight into alcohol's potential role in modifying oestrogen metabolic pathways. Understanding how alcohol influences endogenous metabolic pathways is of high importance given that the biological mechanisms underlying alcohol's strong association with breast and other cancers, which include sex steroid hormone metabolism, remain poorly defined. Alcohol may influence oestrogen metabolism through increased aromatase activity in the liver and other tissues, stimulating conversion of androgens to oestrogens (Rinaldi et al, 2006), decreased catabolism of sex hormones by the liver through accumulation of hepatic nicotinamide-adenine dinucleotide $(\mathrm{NADH})$ - leading to oxidation and inhibition of oestradiol conversion to oestrone (Ginsburg et al, 1996), and direct/indirect adrenal gland cell signalling promotion for DHEAS production (a precursor of oestradiol) (Onland-Moret et al, 2005; Shafrir et al,
2014). How alcohol associates with different oestrogen hydroxylation pathways among postmenopausal women, however, is unknown. A recent analysis evaluated associations of alcohol intake with parent oestrogen/oestrogen metabolites, although women were pre-menopausal and metabolites were measured in urine (Hartman et al, 2016). Alcohol intake was only associated with oestradiol, but not other parent oestrogens/metabolites. Although menopausal status has not been shown to be a significant modifier of the alcohol-breast cancer association (Trentham-Dietz et al, 2014), the majority of breast cancer cases are diagnosed after menopause, with elevated circulating oestrogens being a strong risk factor (Key et al, 2002), highlighting the importance of evaluating alcohol's effects on oestrogen metabolism in this population.

Evaluating ratios of unconjugated to combined oestrogens provides insight into how the pattern of metabolism might vary between never/former and current MHT users. We saw that circulating oestrogen metabolites among MHT users were predominantly conjugated in comparison with non-users. Relative 


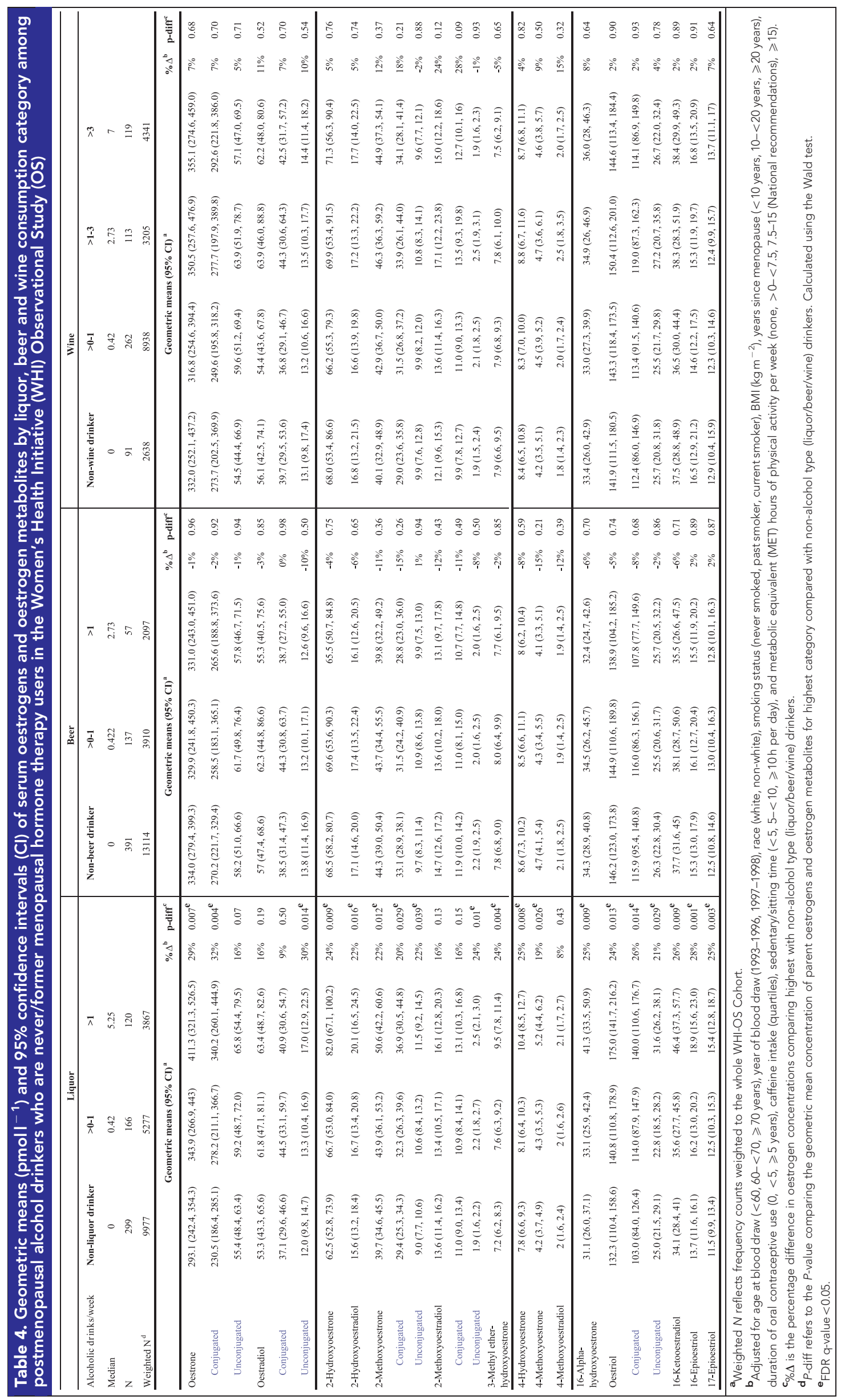


concentration of parent to total oestrogens was also higher, suggesting a potential mechanism for enhanced breast cancer risk with alcohol intake in MHT users. Similar to our observation of an overall alcohol association with parent oestrogens among MHT users, moderate alcohol consumption increased circulating oestradiol among postmenopausal women using MHT but not those who were not on MHT in a randomised, doubleblind, placebo-controlled, crossover feeding study (Ginsburg et al, 1996). Furthermore, among postmenopausal women in a prospective cohort $(N=5035)$, an interaction between intake of two or more alcoholic drinks per day and MHT use was observed for risk of developing breast cancer; drinkers that also used MHT had an increased risk of breast cancer compared with non-MHT users that abstained from alcohol $(\mathrm{HR}=1.27,95 \%$ $\mathrm{CI}=1.09-1.49$ per $1 \mathrm{drink} /$ day increase, $p$-trend $=0.004$ among MHT users; $\mathrm{HR}=0.98,95 \% \mathrm{CI}=0.82-1.78$ per $1 \mathrm{drink} /$ day increase, $p$-trend $=0.79$ among non MHT users) (Nielsen and Gronbaek, 2008). An alcohol-MHT synergistic effect on breast cancer has been supported by analyses within four other large, prospective cohorts (Gapstur et al, 1992; Chen et al, 2002; Suzuki et al, 2005; Zhang et al, 2007). A biological rationale for these findings has been suggested, including decreased conversion of oestrogen to its metabolites, a shift in metabolism to different hydroxylation pathways (Ginsburg et al, 1996), and altered rate of ethinyloestradiol clearance (Ginsburg et al, 1995) among MHT users. In our analysis, drinkers who were taking MHT consumed more total alcohol, on average, than never/former MHT users. It is also possible that dose of alcohol consumption among women in our study may have influenced the differences in magnitudes of association we observed between MHT groups.

We found that highest compared with lowest category of liquor intake among drinkers was associated with $20-30 \%$ increases in both parent oestrogens and 2-, 4-, and 16-hydroxylation pathway metabolites among never/former MHT users. In contrast to total alcohol consumption, never/former MHT users consumed more liquor, on average, than current MHT users. The liquor associations remained with further adjustment for total alcohol intake. When we additionally adjusted for parent oestrogens, the pathway associations were not retained, suggesting that they were not independent of parent oestrogens. This might suggest increased oestrogen formation/reduced catabolism as opposed to hydroxylation pathway effects. The ratio of unconjugated to combined oestrone was nominally reduced with higher liquor consumption, which suggests increased oestrone sulphation or glucuronidation (conversion to oestrone sulphate/glucuronide). Sulphated oestrone is stored as an oestrogen substrate reservoir, whereas glucuronidated oestrone is excreted (Hong and Chen, 2011). Few studies have evaluated associations of sex hormones by types of alcohol, in part due to limitations in sample size (London et al, 1991; Hankinson et al, 1995; Newcomb et al, 1995; Hartman et al, 2016). Feeding studies have generally utilised $95 \%$ ethanol as the alcohol exposure thus have not evaluated wine or beer intake (Dorgan et al, 2001; Mahabir et al, 2004). Further research on differences in sex steroid hormone metabolism by alcohol type is warranted. Polyphenols in red wine have been shown to be a cancer chemopreventive agent (Scalbert et al, 2005). Nonetheless, updated dose-response meta-analyses support that all alcohol types, including red wine intake, are associated with increased risk for colorectal and breast cancers (World Cancer Research Fund/ American Institute for Cancer Research, 2010; World Cancer Research Fund/American Institute for Cancer Research, 2017). While we did not observe an association of wine or beer intake with oestrogen metabolism, it is possible that these alcohol types increase cancer risk through other mechanisms such as formation of acetaldehyde, oxidative stress, altered carcinogen clearance, and impaired immunity.
In the context of cancer prevention, understanding hormonal mechanisms influenced by lifestyle exposures like alcohol consumption may provide targets for cancer prevention intervention in high risk populations. In the same population of women to the current study, parent oestrogens were found to be positively related to endometrial cancer risk (particularly unconjugated oestradiol) (Brinton et al, 2016). Higher oestrone, and 2- and 4- and 16pathway metabolites were also associated with non-serous ovarian cancer risk (Trabert et al, 2016). Our data from the current analysis support current recommendations to limit alcohol consumption for cancer prevention.

Strengths of our study include the high reliability, sensitivity and specificity of the LC-MS/MS assay, comprehensive evaluation of oestrogen/oestrogen metabolites by exogenous hormone use, and the ability to evaluate associations by alcohol type. However, several limitations exist. The median dose of alcohol consumption in our study was modest and may have been inadequate to enable us to detect associations with oestrogen metabolism. Additionally, sample size may have been limited to detect statistically significant associations, given adjustment for multiple comparisons. We were unable to evaluate earlier life alcohol exposure, which may have been higher than postmenopausal consumption. Additionally, the current analysis was cross-sectional at the time of entry into the study. We did not evaluate how alcohol intake changed, or how alcohol intake was associated with oestrogen metabolism over time, which may be relevant in terms of future disease risk. Alcohol was evaluated using a self-reported questionnaire and thus misreporting could lead to alcohol usage misclassification. However, misreporting of intake is not likely to be associated with endogenous hormone concentrations, so associations would likely be attenuated. The analysis was crosssectional so we were unable to make inference about causality. We evaluated usual (12-month) frequency of alcohol consumption, but were unable to compare acute versus chronic alcohol exposure, or alcohol exposure across the life course. We evaluated average weekly alcohol consumption, which may not be the relevant exposure to evaluate effects on oestrogen metabolism since the timing of last alcohol ingestion could influence oestrogen concentration due to the short half-life of oestrogens (Ginsburg et al, 1998). Women enrolled in the WHI-OS included a subset that declined participation in WHI trials, which could have introduced selection bias in terms of alcohol exposure, although participants of the WHI clinical trials (Jackson et al, 2003; Ritenbaugh et al, 2003; Stefanick et al, 2003) had a similar distribution of alcohol consumption to the observational study (Langer et al, 2003). Future studies would also benefit from evaluating adrenal steroid precursors to oestrogens in addition to the analytes evaluated in the current study. We did not have participant genotype data; evaluating gene-alcohol interaction in relation to oestrogen hormone metabolism could contribute to the literature in this area.

In conclusion, this study strengthens the evidence that alcohol consumption increases circulating oestrogen among postmenopausal women. Our observations suggest that alcohol, specifically liquor, influences parent oestrogen concentrations, potentially through increased oestrogen production, as opposed to modifying oestrogen metabolism. Future large, prospective studies with larger average dose of alcohol consumption are needed to further explore alcohol's differential effects on oestrogen metabolism in the 2-, 4-, and 16-hydroxylation pathways.

\section{ACKNOWLEDGEMENTS}

We gratefully acknowledge the members of the Women's Health Initiative Observational study research group and study 
participants. The WHI program is supported by contracts from the National Heart, Lung and Blood Institute, NIH. The authors thank the WHI investigators (JoAnn E. Manson, Barbara V. Howard, Marcia L. Stefanick, Rebecca Jackson, Cynthia A. Thomson, Jean Wactawski-Wende, Marian Limacher, Robert Wallace, Lewis Kuller, Sally Shumaker, Jacques Rossouw, Shari Ludlam, Dale Burwen, Joan McGowan, Leslie Ford, Nancy Geller, Garnet Anderson, Ross Prentice, Andrea LaCroix, Charles Kooperberg) for their dedication, and the study participants for making the programme possible. The WHI programme is funded by the National Heart, Lung, and Blood Institute, National Institutes of Health, U.S. Department of Health and Human Services through contracts HHSN268201100046C, HHSN268201100001C, HHSN 268201100002C, HHSN268201100003C, HHSN268201100004C, and HHSN271201100004C. This study was also supported by the Intramural Research Program of the Division of Cancer Epidemiology and Genetics of the National Cancer Institute.

\section{CONFLICT OF INTEREST}

The authors declare no conflict of interest.

\section{AUTHOR CONTRIBUTIONS}

BT designed the study, oversaw analyses and has primary responsibility for the final content. MCP conducted statistical analysis and wrote the paper. SBC, RP and RTF contributed to the statistical analysis. LAB, NW, and GA contributed to the design and conduct of the original nested case-control studies. GA, LAB, SBC, RTF, SCM, RP, RW, NW and XX provided critical intellectual content to revise the manuscript. All authors reviewed and approved the final manuscript.

\section{REFERENCES}

Benjamini Y, Hochberg Y (1995) Controlling the false discovery rate: a practical and powerful approach to multiple testing. J Roy Stat Soc B 57: 289-300.

Brand JS, Chan MF, Dowsett M, Folkerd E, Wareham NJ, Luben RN, van der Schouw YT, Khaw KT (2011) Cigarette smoking and endogenous sex hormones in postmenopausal women. J Clin Endocrinol Metab 96(10): 3184-3192.

Brinton LA, Trabert B, Anderson GL, Falk RT, Felix AS, Fuhrman BJ, Gass ML, Kuller LH, Pfeiffer RM, Rohan TE, Strickler HD, Xu X, Wentzensen N (2016) Serum estrogens and estrogen metabolites and endometrial cancer risk among postmenopausal women. Cancer Epidemiol Biomarkers Prev 25(7): 1081-1089.

Cavalieri E, Chakravarti D, Guttenplan J, Hart E, Ingle J, Jankowiak R, Muti P, Rogan E, Russo J, Santen R, Sutter T (2006) Catechol estrogen quinones as initiators of breast and other human cancers: implications for biomarkers of susceptibility and cancer prevention. Biochim Biophys Acta 1766(1): $63-78$.

Cavalieri EL, Rogan EG (2016) Depurinating estrogen-DNA adducts, generators of cancer initiation: their minimization leads to cancer prevention. Clin Transl Med 5(1): 12.

Chan MF, Dowsett M, Folkerd E, Bingham S, Wareham N, Luben R, Welch A, Khaw KT (2007) Usual physical activity and endogenous sex hormones in postmenopausal women: the European prospective investigation into cancer-norfolk population study. Cancer Epidemiol Biomarkers Prev 16(5): 900-905.

Chen WY, Colditz G, Rosner B, Hankinson SE, Hunter DJ, Manson JE, Stampfer MJ, Willett WC, Speizer FE (2002) Use of postmenopausal hormones, alcohol, and risk for invasive breast cancer. Ann Intern Med 137(10): 798-804.

Dallal CM, Lacey Jr. JV, Pfeiffer RM, Bauer DC, Falk RT, Buist DS, Cauley JA, Hue TF, LaCroix AZ, Tice JA, Veenstra TD, Xu X, Brinton LA, Group
BaFR (2016) Estrogen metabolism and risk of postmenopausal endometrial and ovarian cancer: the B approximately FIT cohort. Horm Cancer 7(1): 49-64

Dorgan JF, Baer DJ, Albert PS, Judd JT, Brown ED, Corle DK, Campbell WS, Hartman TJ, Tejpar AA, Clevidence BA, Giffen CA, Chandler DW, Stanczyk FZ, Taylor PR (2001) Serum hormones and the alcohol-breast cancer association in postmenopausal women. J Natl Cancer Inst 93(9): $710-715$.

Durrleman S, Simon R (1989) Flexible regression models with cubic splines. Stat Med 8: 551-561.

Friedenreich CM, Woolcott CG, McTiernan A, Ballard-Barbash R, Brant RF, Stanczyk FZ, Terry T, Boyd NF, Yaffe MJ, Irwin ML, Jones CA, Yasui Y, Campbell KL, McNeely ML, Karvinen KH, Wang Q, Courneya KS (2010) Alberta physical activity and breast cancer prevention trial: sex hormone changes in a year-long exercise intervention among postmenopausal women. J Clin Oncol 28(9): 1458-1466.

Ganna A, Lee D, Ingelsson E, Pawitan Y (2014) Rediscovery rate estimation for assessing the validation of significant findings in high-throughput studies. Brief Bioinform 16(4): 563-575.

Gapstur SM, Potter J, Sellers TA, Folsom AR (1992) Increased risk of breast cancer with alcohol consumption in postmenopausal women. Am J Epidemiol 136(10): 1221-1231.

Ginsburg ES, Gao X, Shea BF, Barbieri RL (1998) Half-life of estradiol in postmenopausal women. Gynecol Obstet Invest 45(1): 45-48.

Ginsburg ES, Mello NK, Mendelson JH, Barbieri RL, Teoh SK, Rothman M, Gao X, Sholar JW (1996) Effects of alcohol ingestion on estrogens in postmenopausal women. JAMA 276(21): 1747-1751.

Ginsburg ES, Walsh BW, Shea BF, Gao X, Gleason RE, Barbieri RL (1995) The effects of ethanol on the clearance of estradiol in postmenopausal women. Fertil Steril 63(6): 1227-1230.

Hankinson SE, Willett WC, Manson JE, Hunter DJ, Colditz GA, Stampfer MJ, Longcope C, Speizer FE (1995) Alcohol, height, and adiposity in relation to estrogen and prolactin levels in postmenopausal women. J Natl Cancer Inst 87(17): 1297-1302.

Hartman TJ, Sisti JS, Hankinson SE, Xu X, Eliassen AH, Ziegler R (2016) Alcohol consumption and urinary estrogens and estrogen metabolites in premenopausal women. Horm Cancer 7(1): 65-74.

Hong Y, Chen S (2011) Aromatase, oestrone sulfatase, and 17betahydroxysteroid dehydrogenase: structure-function studies and inhibitor development. Mol Cell Endocrinol 340(2): 120-126.

IARC (2010) IARC monographs on the evaluation of carcinogenic risks to humans. Volume 96. Alcoholic beverage consumption and ethyl carbamate (urethane). IARC: Lyon.

Jackson RD, LaCroix AZ, Cauley JA, McGowan J (2003) The Women's Health Initiative calcium-vitamin D trial: overview and baseline characteristics of participants. Ann Epidemiol 13(9 Suppl): S98-106.

Jefcoate CR, Liehr JG, Santen RJ, Sutter TR, Yager JD, Yue W, Santner SJ, Tekmal R, Demers L, Pauley R, Naftolin F, Mor G, Berstein L (2000) Tissue-specific synthesis and oxidative metabolism of estrogens. $J$ Nat Cancer Inst Monogr 27: 95-112.

Jung S, Wang M, Anderson K, Baglietto L, Bergkvist L, Bernstein L, van den Brandt PA, Brinton L, Buring JE, Heather Eliassen A, Falk R, Gapstur SM, Giles GG, Goodman G, Hoffman-Bolton J, Horn-Ross PL, Inoue M, Kolonel LN, Krogh V, Lof M, Maas P, Miller AB, Neuhouser ML, Park Y, Robien K, Rohan TE, Scarmo S, Schouten LJ, Sieri S, Stevens VL, Tsugane S, Visvanathan K, Wilkens LR, Wolk A, Weiderpass E, Willett WC, ZeleniuchJacquotte A, Zhang SM, Zhang X, Ziegler RG, Smith-Warner SA (2015) Alcohol consumption and breast cancer risk by estrogen receptor status: in a pooled analysis of 20 studies. Int $j$ epidemiol 45(3): 916-928.

Key T, Appleby P, Barnes I, Reeves G, Endogenous H, Breast Cancer Collaborative G (2002) Endogenous sex hormones and breast cancer in postmenopausal women: reanalysis of nine prospective studies. $J$ Natl Cancer Inst 94(8): 606-616.

Key TJ, Appleby PN, Reeves GK, Roddam A, Dorgan JF, Longcope C, Stanczyk FZ, Stephenson Jr HE, Falk RT, Miller R, Schatzkin A, Allen DS, Fentiman IS, Key TJ, Wang DY, Dowsett M, Thomas HV, Hankinson SE, Toniolo P, Akhmedkhanov A, Koenig K, Shore RE, Zeleniuch-Jacquotte A, Berrino F, Muti P, Micheli A, Krogh V, Sieri S, Pala V, Venturelli E, Secreto G, Barrett-Connor E, Laughlin GA, Kabuto M, Akiba S, Stevens RG, Neriishi K, Land CE, Cauley JA, Kuller LH, Cummings SR, Helzlsouer KJ, Alberg AJ, Bush TL, Comstock GW, Gordon GB, Miller SR, Longcope C, Endogenous Hormones Breast Cancer Collaborative G 
(2003) Body mass index, serum sex hormones, and breast cancer risk in postmenopausal women. J Natl Cancer Inst 95(16): 1218-1226.

Key TJ, Appleby PN, Reeves GK, Roddam AW, Helzlsouer KJ, Alberg AJ, Rollison DE, Dorgan JF, Brinton LA, Overvad K, Kaaks R, Trichopoulou A, Clavel-Chapelon F, Panico S, Duell EJ, Peeters PH, Rinaldi S, Fentiman IS, Dowsett M, Manjer J, Lenner P, Hallmans G, Baglietto L, English DR, Giles GG, Hopper JL, Severi G, Morris HA, Hankinson SE, Tworoger SS, Koenig K, Zeleniuch-Jacquotte A, Arslan AA, Toniolo P, Shore RE, Krogh V, Micheli A, Berrino F, Barrett-Connor E, Laughlin GA, Kabuto M, Akiba S, Stevens RG, Neriishi K, Land CE, Cauley JA, Lui LY, Cummings SR, Gunter MJ, Rohan TE, Strickler HD (2011) Circulating sex hormones and breast cancer risk factors in postmenopausal women: reanalysis of 13 studies. Br j cancer 105(5): 709-722.

Kotsopoulos J, Eliassen AH, Missmer SA, Hankinson SE, Tworoger SS (2009) Relationship between caffeine intake and plasma sex hormone concentrations in premenopausal and postmenopausal women. Cancer 115(12): 2765-2774.

Langer RD, White E, Lewis CE, Kotchen JM, Hendrix SL, Trevisan M (2003) The Women's Health Initiative Observational Study: baseline characteristics of participants and reliability of baseline measures. Ann Epidemiol 13(9 Suppl): S107-S121.

Li HL, Gail MH (2012) Efficient adaptively weighted analysis of secondary phenotypes in case-control genome-wide association studies. Hum Hered 73(3): 159-173.

London S, Willett W, Longcope C, McKinlay S (1991) Alcohol and other dietary factors in relation to serum hormone concentrations in women at climacteric. Am J Clin Nutr 53(1): 166-171.

Mahabir S, Baer DJ, Johnson LL, Dorgan JF, Campbell W, Brown E, Hartman TJ, Clevidence B, Albanes D, Judd JT, Taylor PR (2004) The effects of moderate alcohol supplementation on oestrone sulfate and DHEAS in postmenopausal women in a controlled feeding study. Nutr J 3: 11.

Newcomb PA, Klein R, Klein BE, Haffner S, Mares-Perlman J, Cruickshanks KJ, Marcus PM (1995) Association of dietary and life-style factors with sex hormones in postmenopausal women. Epidemiology 6(3): 318-321.

Nielsen NR, Gronbaek M (2008) Interactions between intakes of alcohol and postmenopausal hormones on risk of breast cancer. Int J Cancer 122(5): 1109-1113.

Onland-Moret NC, Peeters PH, van der Schouw YT, Grobbee DE, van Gils CH (2005) Alcohol and endogenous sex steroid levels in postmenopausal women: a cross-sectional study. J Clin Endocrinol Metab 90(3): 1414-1419.

Patterson RE, Kristal AR, Tinker LF, Carter RA, Bolton MP, Agurs-Collins T (1999) Measurement characteristics of the Women's Health Initiative food frequency questionnaire. Ann Epidemiol 9(3): 178-187.

Rinaldi S, Peeters PH, Bezemer ID, Dossus L, Biessy C, Sacerdote C, Berrino F, Panico S, Palli D, Tumino R, Khaw KT, Bingham S, Allen NE, Key T, Jensen MK, Overvad K, Olsen A, Tjonneland A, Amiano P, Ardanaz E, Agudo A, Martinez-Garcia C, Quiros JR, Tormo MJ, Nagel G, Linseisen J, Boeing H, Schulz M, Grobbee DE, Bueno-de-Mesquita HB, Koliva M, Kyriazi G, Thrichopoulou A, Boutron-Ruault MC, Clavel-Chapelon F, Ferrari P, Slimani N, Saracci R, Riboli E, Kaaks R (2006) Relationship of alcohol intake and sex steroid concentrations in blood in pre- and postmenopausal women: the European Prospective Investigation into Cancer and Nutrition. Cancer Causes Control 17(8): 1033-1043.

Ritenbaugh C, Patterson RE, Chlebowski RT, Caan B, Fels-Tinker L, Howard B, Ockene J (2003) The Women's Health Initiative Dietary Modification trial: overview and baseline characteristics of participants. Ann Epidemiol 13(9 Suppl): S87-S97.

Rosner B (1983) Percentage points for a generalized Esd many-outlier procedure. Technometrics 25(2): 165-172.

Sampson JN, Falk RT, Schairer C, Moore SC, Fuhrman BJ, Dallal CM, Bauer DC, Dorgan JF, Shu XO, Zheng W, Brinton LA, Gail MH, Ziegler RG, Xu X, Hoover RN, Gierach GL (2016) Association of estrogen metabolism with breast cancer risk in different cohorts of postmenopausal women. Cancer Res 77(4): 918-925.

Scalbert A, Manach C, Morand C, Remesy C, Jimenez L (2005) Dietary polyphenols and the prevention of diseases. Crit Rev Food Sci Nutr 45(4): 287-306.

Schutze M, Boeing H, Pischon T, Rehm J, Kehoe T, Gmel G, Olsen A, Tjonneland AM, Dahm CC, Overvad K, Clavel-Chapelon F, Boutron-Ruault MC, Trichopoulou A, Benetou V, Zylis D, Kaaks R,
Rohrmann S, Palli D, Berrino F, Tumino R, Vineis P, Rodriguez L, Agudo A, Sanchez MJ, Dorronsoro M, Chirlaque MD, Barricarte A, Peeters PH, van Gils CH, Khaw KT, Wareham N, Allen NE, Key TJ, Boffetta P, Slimani N, Jenab M, Romaguera D, Wark PA, Riboli E, Bergmann MM (2011) Alcohol attributable burden of incidence of cancer in eight European countries based on results from prospective cohort study. Bmj 342: d1584.

Setiawan VW, Haiman CA, Stanczyk FZ, Le Marchand L, Henderson BE (2006) Racial/ethnic differences in postmenopausal endogenous hormones: the multiethnic cohort study. Cancer Epidemiol Biomarkers Prev 15(10): 1849-1855.

Shafrir AL, Zhang X, Poole EM, Hankinson SE, Tworoger SS (2014) The association of reproductive and lifestyle factors with a score of multiple endogenous hormones. Horm Cancer 5(5): 324-335.

Sierksma A, Sarkola T, Eriksson CJ, van der Gaag MS, Grobbee DE, Hendriks HF (2004) Effect of moderate alcohol consumption on plasma dehydroepiandrosterone sulfate, testosterone, and estradiol levels in middle-aged men and postmenopausal women: a diet-controlled intervention study. Alcohol Clin Exp Res 28(5): 780-785.

Sisti JS, Hankinson SE, Caporaso NE, Gu F, Tamimi RM, Rosner B, Xu X, Ziegler R, Eliassen AH (2015) Caffeine, coffee, and tea intake and urinary estrogens and estrogen metabolites in premenopausal women. Cancer Epidemiol Biomarkers Prev 24(8): 1174-1183.

Stefanick ML, Cochrane BB, Hsia J, Barad DH, Liu JH, Johnson SR (2003) The Women's Health Initiative postmenopausal hormone trials: overview and baseline characteristics of participants. Ann Epidemiol 13(9 Suppl): S78-S86.

Suzuki R, Ye W, Rylander-Rudqvist T, Saji S, Colditz G, Wolk A (2005) Alcohol and postmenopausal breast cancer risk defined by estrogen and progesterone receptor status: a prospective cohort study. J Natl Cancer Inst 97(21): 1601-1608.

The Women's Health Initiative Study Group (1998) Design of the Women's Health Initiative clinical trial and observational study. The Women's Health Initiative Study Group. Control Clin Trials 19(1): 61-109.

Trabert B, Brinton LA, Anderson GL, Pfeiffer R, Falk RT, Strickler HD, Sliesoraitis S, Kuller LH, Gass ML, Fuhrman BJ, Xu X, Wentzensen N (2015) Circulating estrogens and postmenopausal ovarian cancer risk in the Women's Health Initiative Observational Study. Cancer Epidemiol Biomarkers Prev 25(4): 648-656.

Trabert B, Brinton LA, Anderson GL, Pfeiffer RM, Falk RT, Strickler HD, Sliesoraitis S, Kuller LH, Gass ML, Fuhrman BJ, Xu X, Wentzensen N (2016) Circulating estrogens and postmenopausal ovarian cancer risk in the Women's Health Initiative Observational Study. Cancer Epidemiol Biomarkers Prev 25(4): 648-656.

Trentham-Dietz A, Sprague BL, Hampton JM, Miglioretti DL, Nelson HD, Titus LJ, Egan KM, Remington PL, Newcomb PA (2014) Modification of breast cancer risk according to age and menopausal status: a combined analysis of five population-based case-control studies. Breast Cancer Res Treat 145(1): 165-175.

World Cancer Research Fund/American Institute for Cancer Research (2010) Continuous Update Project Report. Food, Nutrition, Physical Activity, and the Prevention of Breast Cancer.

World Cancer Research Fund/American Institute for Cancer Research (2017) Continuous Update Project Report: Diet, Nutrition, Physical Activity and Colorectal Cancer.

Yager JD (2015) Mechanisms of estrogen carcinogenesis: the role of E2/E1quinone metabolites suggests new approaches to preventive interventionA review. Steroids 99(Pt A): 56-60.

Zhang SM, Lee I, Manson JE, Cook NR, Willett WC, Buring JE (2007) Alcohol consumption and breast cancer risk in the Women's Health Study. Am J Epidemiol 165(6): 667-676.

This work is published under the standard license to publish agreement. After 12 months the work will become freely available and the license terms will switch to a Creative Commons AttributionNonCommercial-Share Alike 4.0 Unported License.

Supplementary Information accompanies this paper on British Journal of Cancer website (http://www.nature.com/bjc) 